\title{
PRODUCCIÓN, DIGESTIBILIDAD Y RENTABILIDADEN CORDEROS DE DIETAS CON VAINAS DE ACACIA FARNESIANA
}

\author{
PRODUCTION, DIGESTIBILITY AND COST/BENEFIT OF LAMB'S DIETS INCLUDING \\ ACACIA FARNESIANA PODS
}

\author{
Velázquez, A.J. ${ }^{1 \star}$, González, M. ${ }^{1}$, Perezgrovas, R. ${ }^{2}$, Bórquez, J. $^{1}$ y Domínguez, I. ${ }^{1}$
}

\begin{abstract}
${ }^{1}$ Departamento de Nutrición Animal. Facultad de Medicina Veterinaria y Zootecnia. Universidad Autónoma del Estado de México. Instituto Literario número 100. Toluca. Estado de México. México. *jorgevelazqueza@yahoo.com.mx

${ }^{2}$ Instituto de Estudios Indígenas. Universidad Autónoma de Chiapas. Estado de México. México.
\end{abstract}

\section{PALABRAS CLAVE ADICIONALES}

Leguminosas arbóreas. Consumo. Ganancia de peso. Lana. Taninos.

\section{RESUMEN}

Con el objetivo de evaluar el uso de vainas de Acacia farnesiana en la alimentación de ganado lanar se hizo un experimento con corderos de raza Chiapas en crecimiento, que recibieron dietas $(T)$ isoproteínicas con diferentes niveles de inclusión de vainas: $T_{1}=0, T_{2}=100, T_{3}=200, T_{4}=300$ y $T_{5}=400$ $\mathrm{g} / \mathrm{kg}$ (base seca) elaboradas con rastrojo de maíz, harina de soja, salvado de trigo y mezcla de minerales; se estudió la respuesta productiva, digestibilidad y análisis de beneficio-costo. Se utilizaron corderos en confinamiento y alimentación controlada bajo un diseño completamente el azar con cinco tratamientos y cinco repeticiones. El contenido de proteína bruta (PB) de las vainas fue de $115,2 \mathrm{~g} / \mathrm{kg}$ (MS) y de taninos $7,8 \mathrm{~g} / \mathrm{kg}$. El consumo voluntario fue mayor $(p<0,05)$ en $T_{4}$ en relación al grupo control; en tanto la conversión alimenticia y la ganancia diaria de peso fueron similares $(p>0,05)$ entre tratamientos. La producción de lana no mostró diferencias $(p>0,05)$ en peso del vellón sucio y rendimiento al lavado; no obstante, el crecimiento de lana por unidad de superficie $\left(\mathrm{mg} / \mathrm{cm}^{2} / \mathrm{d}\right.$ ía) fue mayor $(\mathrm{p}<0,05)$ con la inclusión de $200 \mathrm{~g} / \mathrm{kg}$ en la dieta. No hubo diferencias de digestibilidad de la materia seca y fibras $(p<0,05)$, mientras que el beneficio-costo con mayor índice fue $\mathrm{T}_{4}$. Se concluye que la inclusión hasta $400 \mathrm{~g} / \mathrm{kg}$ de vainas de Acacia farnesiana en dietas para corderos en crecimiento no disminuye

\section{Additional KEYWORdS}

Legume-trees. Consumption. Weight gain. Wool. Tannins.

la respuesta productiva o la digestibilidad, mientras que con $200 \mathrm{~g} / \mathrm{kg}$ se mejoró el consumo voluntario y el crecimiento de lana, por lo que se puede recomendar su uso para bajar los costos de alimentación.

\section{SUMMARY}

Aiming to evaluate the use of Acacia farnesiana pods in sheep feeding, an experiment was carried out with growing lambs receiving isoproteinic diets $(T)$ with different inclusion levels of pods: $T_{1}=$ $0, T_{2}=100, T_{3}=200, T_{4}=300$ and $T_{5}=400 \mathrm{~g} / \mathrm{kg}$ (dry basis) prepared with maize fodder, soybean meal, wheat bran and minerals mix; productive performance, digestibility and cost-benefit analysis were assessed. Fifty lambs of the Chiapas breed were utilized, kept in closed confinement under a controlled feeding regime, using a completely at random design with five treatments and five repetitions. The crude protein content (CP) of pods was $115.2 \mathrm{~g} / \mathrm{kg}$ (DM) and tannins were $7.8 \mathrm{~g} / \mathrm{kg}$. Voluntary intake was higher $(p<0.05)$ in $T_{4}$ as compared with the control group; feed conversion and average daily gain were similar $(p>0.05)$ among treatments. Wool production showed no per unit area $\left(\mathrm{mg} / \mathrm{cm}^{2} /\right.$ day $)$ was higher $(\mathrm{p}<0.05)$ with the inclusion of $200 \mathrm{~g} / \mathrm{kg}$ of pods in the diet. Dry matter and fiber digestibility showed no 
differences $(p<0.05)$ among treatments, while the cost-benefit ratio was better for $\mathrm{T}_{4}$. It is concluded that the inclusion up to $400 \mathrm{~g} / \mathrm{kg}$ of Acacia farnesiana pods in the diet of growing lambs did not have an effect on their productive response or the digestibility, whereas $200 \mathrm{~g} / \mathrm{kg}$ improved voluntary intake and wool growth, and thus its use may be recommended to lower food costs.

\section{INTRODUCCIÓN}

La identificación de recursos forrajeros regionales a partir de fuentes no convencionales y su evaluación sistemática es una de las premisas centrales del presente trabajo, considerando que pueden ser la base para conformar dietas de bajo costo que refuercen las estrategias de producción pecuaria en sistemas campesinos en pequeña escala, en los cuales los alimentos balanceados comerciales representan los mayores costos (Arriaga-Jordán et al., 2001). En el Estado de Chiapas, al sur de México, la estación seca restringe la disponibilidad de forrajes; no obstante, los ecosistemas regionales ofrecen fuentes alternativas de alimentos susceptibles de ser aprovechados, como son las vainas de Acacia farnesiana que pueden ser utilizadas como fuentes de nutrientes de bajo costo para pequeños rumiantes (Perezgrovas, 1999). Sin embargo, se sabe que esta leguminosa arbustiva contiene taninos, los cuales son agentes antinutricionales (Seigler, 2003) que pueden afectar la respuesta productiva del ganado al disminuir la degradación de la fibra y la utilización de la proteína en el rumen (Min et al., 2003; Frutos et al., 2004). El objetivo de esta investigación fue estudiar el efecto de las vainas de Acacia farnesiana, incorporadas en dietas para corderos en crecimiento, sobre el consumo voluntario, ganancia de peso, producción de lana, digestibilidad y relación beneficiocosto.

\section{MATERIALES Y MÉTODOS}

Material vegetativo. Entre febrero y mayo de 2006 se hizo una recolección a granel de vainas de Acacia farnesiana en predios rurales del municipio de Teopisca, Chiapas ubicados en la región Altos de Chiapas entre los $16^{\circ} 15^{\prime}$ y $17^{\circ} 17^{\prime}$ latitud N, y entre los $91^{\circ} 32^{\prime}$ y $93^{\circ} 35^{\prime}$ ' longitud $O$, a una altitud de 1500 a $2400 \mathrm{~m}$ sobre el nivel del mar. Esta región tiene clima templado subhúmedo, con época de lluvias de junio a noviembre y de secas de diciembre a mayo (Parra, 1989). Las vainas secas se trituraron, a un tamaño de cuatro milímetros, en un molino de martillos marca Dumex, modelo TH4000 y se guardaron en bolsas de plástico. Las vainas molidas se incluyeron en cinco dietas isoproteínicas (T) para corderos en crecimiento (NRC, 2007) a diferentes niveles: $\mathrm{T}_{1}=0, \mathrm{~T}_{2}=100, \mathrm{~T}_{3}=200, \mathrm{~T}_{4}=300 \mathrm{y}_{5}=$ $400 \mathrm{~g} / \mathrm{kg}$ (bs), mezcladas con rastrojo de maíz, harina de soja, salvado de trigo y minerales. Los valores del análisis químico de dietas e ingredientes se tomaron de un

Tabla I. Composición química ( $\mathrm{g} / \mathrm{kg} \mathrm{MS}$ ) de los ingredientes utilizados en las dietas experimentales. (Chemical composition ( $\mathrm{g} / \mathrm{kg} \mathrm{DM}$ ) of feedstuffs utilized in experimental diets).

\begin{tabular}{lcccccccc}
\hline Concepto & MS & PC & FDN & FDA & LAD & CEN & EE & TAN \\
\hline Vainas de A. farnesiana & 896,0 & 115,2 & 476,0 & 373,2 & 134,0 & 37,7 & 17,9 & 7,8 \\
Rastrojo de maíz & 898,2 & 57,4 & 658,7 & 397,0 & 114,0 & 32,0 & 27,1 & - \\
Soya & 901,2 & 476,7 & 176,5 & 74,2 & 59,0 & 63,8 & 21,3 & - \\
Salvado & 892,5 & 157,5 & 508,9 & 188,3 & 157,4 & 54,2 & 38,9 & - \\
\hline
\end{tabular}

TAN = Taninos. 
estudio previo y se presentan en la tabla I (Velázquez et al., 2011).

Animales y alimentación. Se utilizaron 50 corderos de la raza Chiapas con peso inicial de 8,9 $\pm 1,3 \mathrm{~kg}$ asignados en forma aleatoria a uno de cinco tratamientos. Las unidades experimentales se conformaron con los animales alojados en pares en 25 corraletas de madera dentro de una galera techada por un periodo experimental de 99 días. El alimento se ofreció en dos ocasiones $(8: 00$ y 15:00 h) tras un periodo de adaptación de 7 días; así también tuvieron agua a libre acceso y se incluyeron sales minerales mezcladas en las dietas experimentales. Los animales se desparasitaron en forma rotativa utilizando tres productos a intervalo mensual: closantel (closantil, chinoin, $2 \mathrm{ml} / 10 \mathrm{~kg}$ de peso vivo), albendazole (valbazen, pfizer, $1 \mathrm{ml} / 10 \mathrm{~kg}$ de peso vivo), y abamectina (génesis LA, bio zoo, 1ml/50 kg de peso vivo).
Respuesta productiva. El consumo voluntario se midió registrando diariamente el alimento ofrecido y rechazado (Allen, 1996). Los corderos se pesaron cada quince días en ayuno por las mañanas. El cálculo de la conversión alimenticia se hizo dividiendo el consumo entre la ganancia de peso (Minson, 1990). En la producción de lana se estudió la longitud de la mecha, peso del vellón sucio, crecimiento de lana por unidad de superficie y calidad del vellón. Para establecer la longitud del mechón, se tomaron muestras de lana de la parte media lateral izquierda de los animales y se guardaron en una tarjeta de cartulina doblada a lo largo. Las muestras se procesaron siguiendo técnicas estandarizadas (Perezgrovas et al., 1997).

A los 37 días de iniciado el experimento se realizó la trasquila con esquiladora mecánica, y se pesó el vellón sucio. Cinco semanas después se obtuvo la primera muestra para los estudios de crecimiento de lana,

Tabla II. Composición química ( $\mathrm{g} / \mathrm{kg} \mathrm{MS}$ ) de tratamientos $(\mathrm{T})$ con diferentes niveles de inclusión de vainas de Acacia farnesiana. (Chemical composition (g/kg MS) of treatments $(\mathrm{T})$ with different levels of inclusion of Acacia farnesiana pods).

\begin{tabular}{lccccc}
\hline Ingrediente / Inclusión & $\mathrm{T}_{1}$ & $\mathrm{~T}_{2}$ & $\mathrm{~T}_{3}$ & $\mathrm{~T}_{4}$ & $\mathrm{~T}_{5}$ \\
\hline Vainas de Acacia farnesiana & 0.0 & 100 & 200 & 300 & 400 \\
Rastrojo de maíz & 795 & 710 & 624 & 538 & 450 \\
Harina de soja & 130 & 115 & 101 & 87 & 75 \\
Salvado de trigo & 50 & 50 & 50 & 50 & 50 \\
Minerales $^{1}$ & 25 & 25 & 25 & 25 & 25 \\
Análisis calculado & & & & & \\
Materia seca (MS) & 910 & 903 & 914 & 914 & 917 \\
Proteína bruta (PC) & 117 & 119 & 113 & 113 & 116 \\
Fibra detergente neutro (FDN) & 540 & 507 & 562 & 561 & 543 \\
Energía metabolizable * (EM, MJ/kg) & 9,39 & 8,93 & 9,09 & 8,44 & 8,13 \\
Cenizas & 53 & 55,5 & 57,3 & 56,4 & 60 \\
Calcio (Ca) & 6,5 & 6,5 & 6,5 & 6,5 & 6,5 \\
Fósforo (P) & 4,5 & 4,5 & 4,5 & 4,5 & 4,5 \\
Taninos (TAN) & 0,05 & 1,2 & 2,2 & 2,9 & 3,6 \\
\hline
\end{tabular}

*EM datos obtenidos de Velázquez et al. (2007).

$T_{1}=$ Control; $T_{2}=100 \mathrm{~g} / \mathrm{kg}$ vainas; $T_{3}=200 \mathrm{~g} / \mathrm{kg}$ vainas; $T_{4}=300 \mathrm{~g} / \mathrm{kg}$ vainas; $T_{5}=400 \mathrm{~g} / \mathrm{kg}$ vainas.

${ }^{1}$ Minerales incluidos: $\mathrm{Ca}=15 \% ; \mathrm{P}=7 \% ; \mathrm{Mg}=0,25 \% ; \mathrm{Zn}=0,45 \% ; \mathrm{Cu}=0,05 \% ; \mathrm{Co}=0,014 \% ; \mathrm{Fe}=0,35 \%$; Yodo $=0,001 \%$. 
con máquina trasquiladora realizando un corte sobre la parte media lateral derecha en una superficie aproximada de $100 \mathrm{~cm}^{2}$, colocando al animal en decúbito lateral. La superficie recortada se midió con una precisión de $0,5 \mathrm{~cm}^{2}$ y se registró en tarjetas de control. La segunda muestra se obtuvo cinco semanas más tarde haciendo un corte sobre la superficie del primer muestreo con la misma técnica. Las muestras individuales se identificaron y se colocaron en bolsas de papel hasta su procesamiento en el laboratorio. La calidad del vellón fue determinada por grupos de mujeres indígenas dos días antes de la trasquila con base en sus conocimientos tradicionales con un método tacto-visual que emplea dedos y nudillos (Perezgrovas, 1998), el cual ha sido estandarizado y forma parte de las variables evaluadas por el programa de mejoramiento genético del borrego Chiapas (Perezgrovas, 2005).

Prueba de digestibilidad. Tres días antes de finalizar el experimento, se obtuvieron muestras de heces del $50 \%$ de los corderos y se congelaron a $-4^{\circ} \mathrm{C}$ hasta su posterior análisis. La digestibilidad se determinó según la metodología propuesta
Tabla III. Efecto de dietas con diferentes niveles de inclusión de vainas de Acacia farnesiana sobre el consumo voluntario $(C V)$, ganancia diaria de peso (GDP) y conversión alimenticia (CA) de corderos en crecimiento. (Effect of diets with different inclusion levels of Acacia farnesiana pods on voluntary consumption (CV), weight gain (GDP) and feeding efficiency (CA) in growing lambs).

\begin{tabular}{lccc} 
Tratamiento CV (g/día MS) & GDP (g) & CA (kg) \\
\hline $\mathrm{T}_{1}$ & $570,4 \pm 38,8^{\mathrm{b}}$ & $67,2 \pm 7,8$ & $7,96 \pm 0,88$ \\
$\mathrm{~T}_{2}$ & $570,4 \pm 27,5^{\mathrm{b}}$ & $74,8 \pm 7,2$ & $7,14 \pm 0,75$ \\
$\mathrm{~T}_{3}$ & $645,6 \pm 65,8^{\text {ab }}$ & $77,8 \pm 7,6$ & $7,73 \pm 0,69$ \\
$\mathrm{~T}_{4}$ & $652,8 \pm 86,6^{\mathrm{a}}$ & $75,8 \pm 14,5$ & $8,16 \pm 1,36$ \\
$\mathrm{~T}_{5}$ & $614,6 \pm 50,40^{\text {ab }}$ & $70,2 \pm 9,6$ & $8,26 \pm 1,32$ \\
EEM & 3,39 & 1,39 & 0,45 \\
\hline
\end{tabular}

abMedias con distinta literal en la misma columna, son diferentes $(p<0,05)$.

$\mathrm{EEM}=$ error estándar de la media.

$\mathrm{T}_{1}=0 ; \mathrm{T}_{2}=100 ; \mathrm{T}_{3}=200 ; \mathrm{T}_{4}=300 ; \mathrm{T}_{5}=400 \mathrm{~g} / \mathrm{kg} \mathrm{de}$ vainas.

por Van Keulen y Young (1977), mediante la determinación de cenizas insolubles en detergente ácido, utilizando $0,5 \mathrm{~g}$ de muestras de heces y alimento. Los datos fueron ajus-

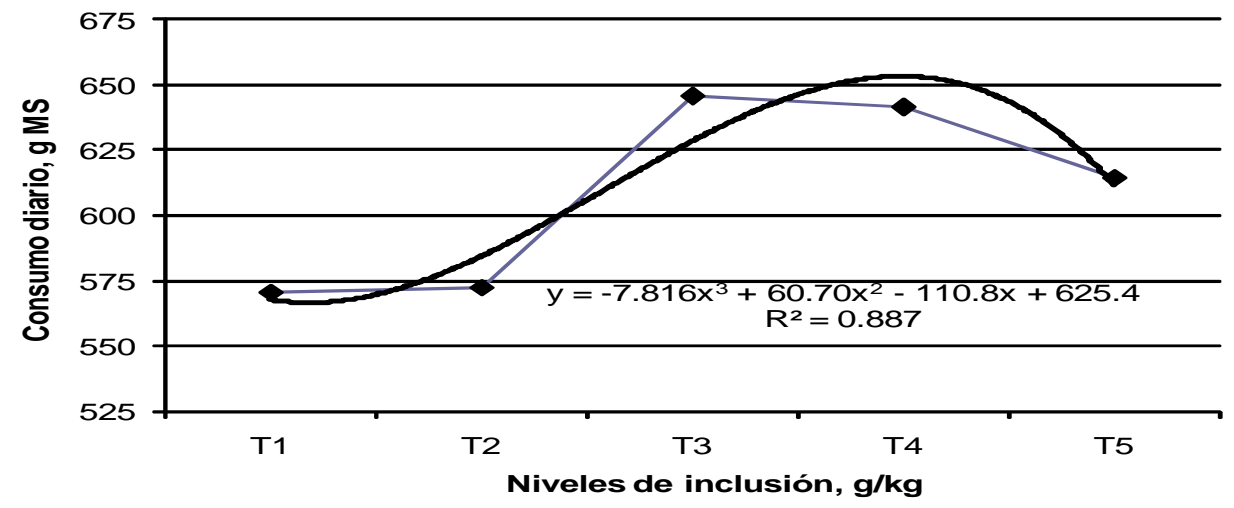

Figura 1. Análisis de regresión del consumo voluntario en corderos alimentados con dietas con diferentes niveles de inclusión de vainas de Acacia farnesiana. $T_{1}=$ control; $T_{2}=100 \mathrm{~g} /$ $\mathrm{kg} ; T_{3}=200 \mathrm{~g} / \mathrm{kg} ; T_{4}=300 \mathrm{~g} / \mathrm{kg} ; T_{5}=400 \mathrm{~g} / \mathrm{kg}$. (Regression analysis of voluntary consumption in lambs supplemented with diets with different inclusion levels of Acacia farnesiana pods. $T_{1}=$ control; $T_{2}=100 \mathrm{~g} / \mathrm{kg} ; T_{3}=200 \mathrm{~g} / \mathrm{kg} ; \mathrm{T}_{4}=300 \mathrm{~g} / \mathrm{kg} ; \mathrm{T}_{5}=400 \mathrm{~g} / \mathrm{kg}$ ). 
tados por las ecuaciones propuestas por Faichney (1975):

$$
\begin{aligned}
& E F=C V * C I D A \text { (alimento) / CIDA (heces) } \\
& D=C V-E F / C V
\end{aligned}
$$

\section{Donde:}

$E F=$ excreción fecal (kg/día);

$\mathrm{CV}=$ consumo voluntario ( $\mathrm{kg} / \mathrm{día})$;

$\mathrm{CIDA}=$ cenizas insolubles en detergente ácido

$(\mathrm{g} / \mathrm{kg})$;

$\mathrm{D}=$ digestibilidad $(\mathrm{g} / \mathrm{kg})$.

Análisis beneficio-costo. Se cuantificó el costo de la mano de obra por corte y acarreo de vainas de Acacia farnesiana y el costo de los diferentes insumos (rastrojo de maíz, soja, salvado y sales minerales). Para efectos de cálculo, se asignó a cada ingrediente el costo comercial actualizado en la región, aun cuando fueran producidos en las propias instalaciones. El valor de medicamentos, otros materiales y mano de obra asociados al manejo de los corderos se asumió como costo fijo. El índice de la rela-

Tabla IV. Digestibilidad de la materia seca (MS) y fibra detergente neutro (FDN) en corderos alimentados con diferentes niveles (T) de inclusión de vainas de Acacia farnesiana. (Digestibility of dry matter (MS) and detergent neutral fiber (FDN) in lambs fed with different inclusion levels of Acacia farnesiana pods).

\begin{tabular}{lcc}
\hline Tratamientos & \multicolumn{2}{c}{$\begin{array}{c}\text { Digestibilidad } \\
(\mathrm{g} / \mathrm{kg})\end{array}$} \\
& $\mathrm{MS}$ & FDN \\
\hline $\mathrm{T}_{1}$ & $528,4 \pm 66,7$ & $423,1 \pm 43,9$ \\
$\mathrm{~T}_{2}$ & $518,6 \pm 42,5$ & $426,3 \pm 53,8$ \\
$\mathrm{~T}_{3}$ & $518,3 \pm 37,9$ & $404,1 \pm 55,6$ \\
$\mathrm{~T}_{4}$ & $516,0 \pm 24,9$ & $433,7 \pm 26,8$ \\
$\mathrm{~T}_{5}$ & $516,3 \pm 14,4$ & $425,1 \pm 8,23$ \\
EEM & 16,69 & 16,69 \\
\hline
\end{tabular}

EEM $=$ error estándar de la media.

$\mathrm{T}_{1}=0 ; \mathrm{T}_{2}=100 ; \mathrm{T}_{3}=200 ; \mathrm{T}_{4}=300 ; \mathrm{T}_{5}=400 \mathrm{~g} / \mathrm{kg} \mathrm{de}$ vainas.

FDN= Fibra detergente neutro. ción beneficio-costo se obtuvo a partir de la suma total de beneficios por venta de lana y corderos en pie, sustrayendo la suma total de costos de producción multiplicada por un factor de actualización del 8,5\% que corresponde a la inflación reportada por el banco de México en el presente año.

Análisis estadístico. El diseño experimental fue completamente al azar con cinco tratamientos y cinco repeticiones (Steel y Torrie, 1980). Se hizo un análisis de regresión para establecer las ecuaciones, los coeficientes de determinación $\left(\mathrm{R}^{2}\right)$ para cada una de las variables y la significancia de la prueba. En las variables con significancia $(\mathrm{p}<0,1)$ se realizó una prueba de medias (Fisher) para ubicar las diferencias y se establecieron líneas de tendencia polinomiales de tercer orden. Los datos fueron procesados con la paquetería minitab V 10.51.

\section{RESULTADOSYDISCUSIÓN}

El análisis químico proximal (BS) de las vainas de Acacia farnesiana mostró que contienen 115,2 g/kg de PB, 6,82 ml/kg de EM, 896,0 g/kg de MS, 476,0 g/kg de FDN y $7,8 \mathrm{~g} / \mathrm{kg}$ de taninos (tabla I). El contenido nutrimental de los tratamientos incluidos la concentración de taninos se registra en la tabla II. Se observó que la proteína aportada por las vainas de Acacia farnesiana contribuyó a la proteína bruta de los tratamientos con más del doble de la aportada por el rastrojo de maíz, sustituyendo también una parte importante de la suministrada por la soja.

La respuesta productiva no se vio afectada al utilizar el máximo nivel de vainas en la dieta (400 g/kg) (tabla III). Se observó que el consumo voluntario en $\mathrm{T}_{4}$ fue mayor $(\mathrm{p}<0,05)$ que el tratamiento control (figura 1). García et al. (2009) reportaron no haber encontrado diferencias en el consumo de dietas a base de vainas de Acacia farnesiana con niveles entre 120 y $240 \mathrm{~g} / \mathrm{kg}$ en borregos de pelo; no obstante, otros autores mencionan incrementos en el consumo con dietas 
Tabla $\mathbf{V}$. Efecto de dietas con diferentes niveles de inclusión de vainas de Acacia farnesiana sobre la calidad del vellón (Ca) y longitud de la mecha en fibras largas (FL), fibras cortas (FC) y fibra kemp (K) en corderos en crecimiento. (Effect of diets with different inclusion levels of Acacia farnesiana pods on fleece quality (Ca) and staple length in large fiber (FL), short fiber (FC) and kemp fiber $(K)$ in growing lambs).

\begin{tabular}{lcccc}
\hline Tratamiento & $\mathrm{Ca}(1-4)^{\star}$ & $\mathrm{FL}(\mathrm{cm})$ & $\mathrm{FC}(\mathrm{cm})$ & $\mathrm{K}(\mathrm{cm})$ \\
\hline $\mathrm{T}_{1}$ & $3,56 \pm 0,33$ & $8,60 \pm 0,98$ & $6,20 \pm 0,32$ & $0,10 \pm 0,22$ \\
$\mathrm{~T}_{2}$ & $3,42 \pm 0,26$ & $8,45 \pm 0,65$ & $6,45 \pm 0,82$ & $0,60 \pm 0,84$ \\
$\mathrm{~T}_{3}$ & $3,40 \pm 0,26$ & $8,85 \pm 1,69$ & $6,15 \pm 0,74$ & $0,40 \pm 0,65$ \\
$\mathrm{~T}_{4}$ & $3,34 \pm 0,33$ & $7,90 \pm 0,80$ & $6,00 \pm 0,61$ & $0,60 \pm 0,98$ \\
$\mathrm{~T}_{5}$ & $3,52 \pm 0,28$ & $8,45 \pm 0,57$ & $6,15 \pm 0,62$ & $0,15 \pm 0,33$ \\
EEM & 0,24 & 0,45 & 0,24 & 0,19 \\
\hline
\end{tabular}

*Escala 1= pobre, 2= regular, 3= buena, 4= excelente (Perezgrovas, 1990).

$\mathrm{EEM}=$ error estándar de la media.

$T_{1}=0 ; T_{2}=100 ; T_{3}=200 ; T_{4}=300 ; T_{5}=400 \mathrm{~g} / \mathrm{kg}$ de vainas.

incluyendo hasta $450 \mathrm{~g} / \mathrm{kg}$ de vainas en ovinos (Kibon y Mainá, 1993). La ganancia diaria de peso y la conversión alimenticia no mostraron diferencias ( $\mathrm{p}>0,05)$ entre tratamientos, lo cual coincide con otros estudios similares como los realizados por Karim et al. (2004) y Sen et al. (2002), en los que tampoco se halló un efecto sobre los indicadores de crecimiento. Sin embargo, otros autores reportaron una menor ganancia de peso en corderos alimentados con vainas de Acacia farnesiana en dietas con $120 \mathrm{~g} / \mathrm{kg}$ de vainas (10 g/kg de taninos) (García et al., 2009). Por su parte, Barry

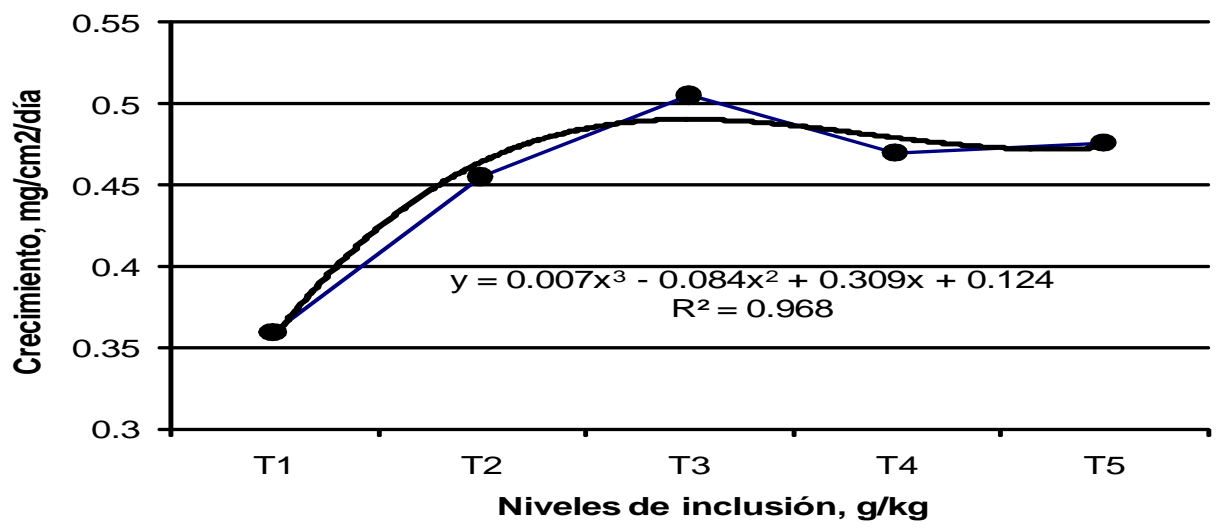

Figura 2. Análisis de regresión del crecimiento de lana por unidad de superficie en corderos alimentados con dietas con diferentes niveles de inclusión de vainas de Acacia farnesiana. $T_{1}=$ control; $T_{2}=100 \mathrm{~g} / \mathrm{kg} ; T=200 \mathrm{~g} / \mathrm{kg} ; T_{4}=300 \mathrm{~g} / \mathrm{kg} ; T_{5}=400 \mathrm{~g} / \mathrm{kg}$. (Regression analysis of wool growth per unit area in lambs fed with diets with different inclusion levels of Acacia farnesiana pods. $T_{1}=$ control; $T_{2}=100 \mathrm{~g} / \mathrm{kg} ; T_{3}=200 \mathrm{~g} / \mathrm{kg} ; T_{4}=300 \mathrm{~g} / \mathrm{kg} ; T_{5}=400 \mathrm{~g} / \mathrm{kg}$ ). 
Tabla VI. Efecto de dietas con diferentes niveles de inclusión de vainas de Acacia farnesiana sobre el peso del vellón sucio (PVS), y crecimiento de lana por unidad de superficie (CLUS) en corderos en crecimiento. (Effect of diets with different inclusion levels of Acacia farnesiana pods on greasy fleece weight (PVS), and wool growth per unit area (CLUS) in growing lambs).

\begin{tabular}{lccc}
\hline Tratamiento & $\begin{array}{c}\text { PVS } \\
\mathrm{g}\end{array}$ & $\begin{array}{c}\text { CLUS (4 de junio) } \\
\mathrm{mg} / \mathrm{cm}^{2} / \mathrm{día}\end{array}$ & $\begin{array}{c}\text { CLUS (4 de julio) } \\
\mathrm{mg} / \mathrm{cm}^{2} / \mathrm{dí}^{\mathrm{a}}\end{array}$ \\
\hline $\mathrm{T}_{1}$ & $249,8 \pm 25,0$ & $0,477 \pm 0,12$ & $0,359 \pm 0,10^{\mathrm{b}}$ \\
$\mathrm{T}_{2}$ & $222,2 \pm 45,3$ & $0,526 \pm 0,14$ & $0,455 \pm 0,05^{\mathrm{ab}}$ \\
$\mathrm{T}_{3}$ & $233,4 \pm 64,6$ & $0,505 \pm 0,11$ & $0,505 \pm 0,03^{\mathrm{a}}$ \\
$\mathrm{T}_{4}$ & $231,8 \pm 44,6$ & $0,477 \pm 0,08$ & $0,470 \pm 0,10^{\mathrm{ab}}$ \\
$\mathrm{T}_{5}$ & $263,2 \pm 44,7$ & $0,570 \pm 0,13$ & $0,476 \pm 0,11^{\mathrm{a}}$ \\
EEM & 3,05 & 0,15 & 0,13 \\
& & & \\
\hline
\end{tabular}

${ }^{a b}$ Medias con distinta literal en la misma columna son diferentes $(p<0,05)$.

EEM = error estándar de la media.

$\mathrm{T}_{1}=0 ; \mathrm{T}_{2}=100 ; \mathrm{T}_{3}=200 ; \mathrm{T}_{4}=300 ; \mathrm{T}_{5}=400 \mathrm{~g} / \mathrm{kg}$ de vainas.

(1985) reportó una reducción importante en la ganancia de peso en corderos alimentados con Lotus pedunculatus, con una dieta que contenía 76 a 96 g/kg MS de taninos.

La explicación de las diferencias, con relación a los resultados del presente estudio, posiblemente se deba a que los corderos chiapas empleados en este trabajo son considerados una raza autóctona con características primitivas (Perezgrovas, 2005), por lo que pueden estar mejor adaptados al consumo de alimentos con alto contenido de taninos (Barry, 1985; Silanikove et al., 1996); también se puede pensar que la concentración de taninos en las dietas no haya sido suficiente para afectar negativamente el consumo, ya que los niveles incluidos en los tratamientos son menores al rango de consumo considerado por otros autores como aceptable, de 22 a $50 \mathrm{~g} / \mathrm{kg}$ por día (Kumar y Vaithiyanathan, 1990; Min et al., 2003).

La digestibilidad de la materia seca y fibra detergente neutra no mostró diferencias $(\mathrm{p}>0,05)$ entre tratamientos (tabla IV). En contraste, McSweeney et al. (2005) reportaron una disminución de la digestibilidad in vitro de nutrientes por efecto de los taninos; es posible que estas diferencias se deban a los distintos niveles de concentración de taninos utilizados en las dietas. Por otro lado, es importante señalar que en esta investigación no se observó intoxicación por el uso de vainas hasta en $400 \mathrm{~g} / \mathrm{kg}$ de inclusión, a diferencia de otros trabajos (Nantoumé et al., 2001) en los que se encontró que el consumo excesivo de hojas de Acacia berlandieri y heno de alfalfa (75:25) produjo ataxia de los miembros posteriores en caprinos angora; en el mismo sentido, Hervás et al. (2003) mencionaron signos de intoxicación en borregas canuladas alimentadas con 166 g/kg de taninos. Estas diferencias probablemente se deban a que la concentración de tanino en las dietas con Acacia farnesiana en esta investigación no fueron tan altas, además de no haber utilizado las hojas sino las vainas.

Los resultados de calidad del vellón y longitud de la mecha se presentan en la tabla $\mathbf{V}$, observándose que no hay diferencias $(p>0,05)$ entre tratamientos, de la misma manera que sucedió para el peso del vellón sucio y crecimiento de lana del primer muestreo (tabla VI); sin embargo, el crecimiento por unidad de superficie ( $\mathrm{mg} / \mathrm{cm}^{2} /$ día) en el segundo muestreo mostró ser mayor en $\mathrm{T}_{3}$ $(p<0,05)$ que el grupo testigo (505 vs. $359 \mathrm{mg} /$ 
$\mathrm{cm}^{2} /$ día) (figura 2). Estos resultados coinciden con los reportes de Perezgrovas y Castro (2000b), quienes indican que los parámetros de crecimiento de lana para la raza Chiapas, que producen en condiciones de alta marginación en México, van de 0,37 $\pm 0,05$ a $0,46 \pm 0,06 \mathrm{~g} / \mathrm{cm}^{2} /$ día en ovejas adultas en pastoreo, así como con Velazquez etal.(2005), que reportan un crecimiento de lana de $0,39 \pm$ $0,08 \mathrm{~g} / \mathrm{cm}^{2} /$ día en corderos en crecimiento en pastoreo y suplementados con vainas de Acacia farnesiana y rastrojo de maíz.

Por otro lado, resulta difícil comparar los resultados de calidad de lana con los reportes de literatura debido a que no hay una equivalencia en el concepto de calidad de lana. Algunos autores (Black y Reis, 1979; Arbiza y De Lucas, 1997), indicaron que la calidad está representada por el diámetro o finura de las fibras; mientras que según Perezgrovas y Castro (2000a), en los sistemas tradicionales de cría de ganado lanar en los altos de chiapas los principales criterios de calidad son la longitud de la mecha y la proporción de fibras largas.

Finalmente la evaluación de la relación beneficio-costo mostró que $\mathrm{T}_{4}$ fue mejor que el grupo control (tabla VII). Los resultados de este estudio coinciden con lo reportado por Velázquez et al. (2005), quienes

Tabla VII. Relación beneficio-costo de tratamientos (T) con diferentes niveles de inclusión de vainas de Acacia farnesiana en corderos. (Cost-benefit ratio of treatments $(T)$ with different levels of inclusion of Acacia farnesiana pods in lambs).

\begin{tabular}{lccccccc}
\hline Tratamiento & Ingresos & $\begin{array}{c}\text { Factor } \\
\text { actual }(8,5 \%)\end{array}$ & $\begin{array}{c}\text { Ingresos } \\
\text { actualizados }\end{array}$ & $\begin{array}{c}\text { Costos } \\
\text { Costos totales }\end{array}$ & $\begin{array}{c}\text { Factor } \\
\text { actualizados }\end{array}$ & RBC \\
\hline $\mathrm{T}_{1}$ & $\$ 2545,88$ & 0,922 & $\$ 2346,43$ & $\$ 1689,21$ & 0,922 & $\$ 1556,87$ & 1,51 \\
$\mathrm{~T}_{2}$ & $\$ 2580,75$ & 0,922 & $\$ 2378,57$ & $\$ 1682,48$ & 0,922 & $\$ 1550,67$ & 1,53 \\
$\mathrm{~T}_{3}$ & $\$ 2649,75$ & 0,922 & $\$ 2442,17$ & $\$ 1722,05$ & 0,922 & $\$ 1587,15$ & 1,54 \\
$\mathrm{~T}_{4}$ & $\$ 2678,63$ & 0,922 & $\$ 2468,78$ & $\$ 1725,38$ & 0,922 & $\$ 1590,21$ & 1,55 \\
$\mathrm{~T}_{5}$ & $\$ 2542,50$ & 0,922 & $\$ 2343,32$ & $\$ 1696,21$ & 0,922 & $\$ 1563,33$ & 1,50 \\
\hline
\end{tabular}

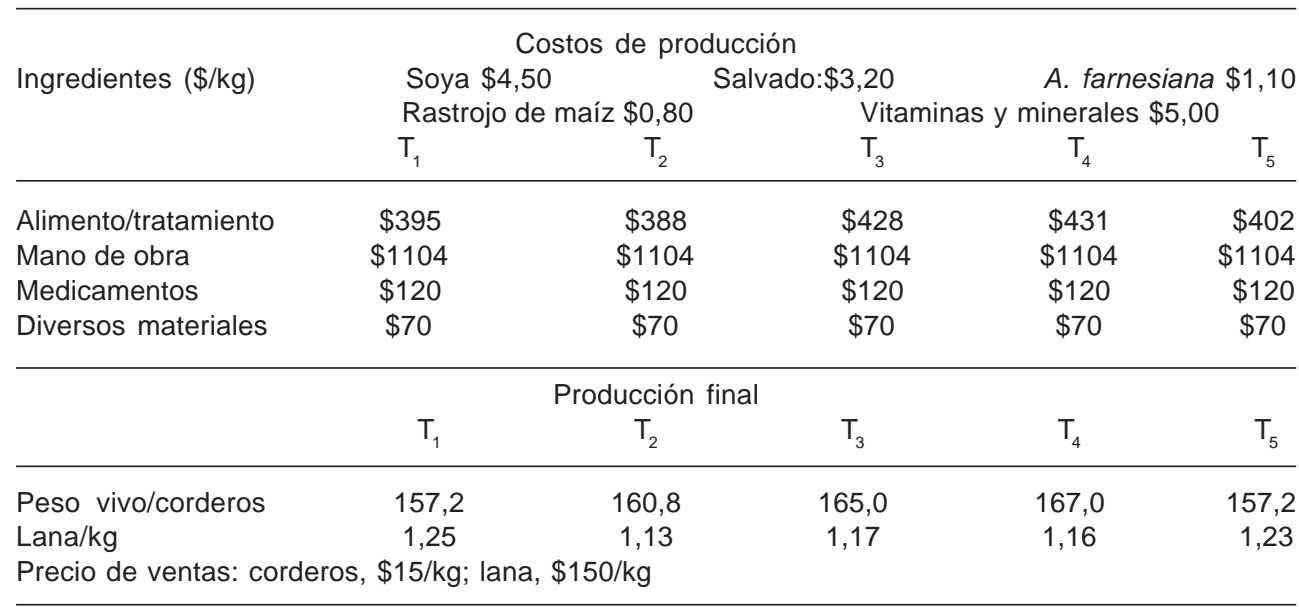

$T_{1}=0 \mathrm{~g} / \mathrm{kg}$ de vainas; $T_{2}=100 \mathrm{~g} / \mathrm{kg}$ de vainas; $T_{3}=200 \mathrm{~g} / \mathrm{kg}$ de vainas; $T_{4}=300 \mathrm{~g} / \mathrm{kg}$ de vainas; $T_{5}=400$ $\mathrm{g} / \mathrm{kg}$ de vainas.

Archivos de zootecnia vol. 60, núm. 231, p. 486. 


\section{DIETAS PARA CORDERO CON VAINAS DE ACACIA FARNESIANA}

encontraron una mayor relación beneficiocosto en dietas que incluyeron $200 \mathrm{~g} / \mathrm{kg}$ de vainas de Acacia farnesiana y rastrojo de maíz para corderos en crecimiento en condiciones de pastoreo. Es posible que estas diferencias se deban al aporte de proteína bruta de las vainas de Acacia farnesiana que al sustituir al de la soja contribuyó a reducir costos debido a su menor precio, pero también a que el impacto de los taninos de las vainas no influyó negativamente en la respuesta productiva.

\section{BIBLIOGRAFÍA}

Allen, M. 1996. Physical constraints on voluntary intake of forages by ruminants. J. Anim. Sci., 74: 3063-3075.

Arbiza, A.S. y De Lucas, T.J. 1997. Lana, producción y características. UAEM. México.

Arriaga-Jordán, C., Flores-Gallegos, F., PeñaCarmona, B., Albarrán-Portillo, A., García-Martínez, A., Espinosa-Ortega, C., GonzálezEsquivel y Castelán-Ortega, O. 2001. Participatory on-farm evaluation of the response to concentrate feeding supplementation by cows in early lactation in smallholder peasant dairy production systems in the highlands of central Mexico. J. Agric. Sci., 137: 97-103.

Barry, T.N. 1985. The role of condensed tannins in the nutritional value of Lotus pedunculatus for sheep. Brit. J. Nutr., 54: 211-217.

Black, J.L. and Reis, P.J. 1979. Physiological and environmental limitations to wool growth. C.S.I.R.O. Sydney. Australia.

Faichney, G.J. 1975. The use of markers to partition digestion within the gastrointestinal tract of ruminants. In: I.W. McDonald and A.C. Warner (Eds.). Digestion and metabolism in rumiants. University of New England Publishing Unit. Armidale. Australia. pp. 277-291.

Frutos, P., Hervás, G., Giradles, F.J. and Mantecón, R. 2004. Tannins and ruminant nutrition. Span. J. Agric. Res., 2: 191-202.

García W.R., Goñi, C.S., Olguin, P.A., Diaz, S.G. and Arriaga, J.C. 2009. Huizache (Acacia farnesiana) whole pods (flesh and seeds) as an alternative feed for sheep in Mexico. Trop.

\section{CONCLUSIONES}

La inclusión de 200 a 400 g/kg (bs) de vainas de la leguminosa arbórea Acacia farnesiana en dietas para ovinos puede ser recomendable, ya que mejora el consumo y no influye negativamente en la respuesta productiva de los animales ni en la producción de lana. Por su menor costo y por su inocuidad en los niveles de inclusión estudiados, es factible utilizar este recurso regional como parte de las estrategias de alimentación del ganado lanar.

Anim. Health Prod. DOI 10.1007/s11250-0099355-2.

Hervás, G., Frutos, P., Giráldez, F., Mantecón, A. and Álvarez del Pino, M. 2003. Effect of different doses of quebracho tannins extract on rumen fermentation in ewes. Anim. Feed Sci. Techn., 109: 65-78.

Karim, S. A., Mehta, B.S., Sureshkumar, S. and Verma, D.L. 2004. Growth performance and carcass traits of Bharat Merino lambs maintained under intensive feeding and grazing with supplementation. Indian J. Anim. Sci., 74: 977-979.

Kumar, R. and Vaithiyanathan, S. 1990. Occurrence, nutritional significance and effect on animal productivity of tannins in tree leaves. Anim. Feed Sci. Techn., 30: 21-38.

Kibon, A. y Mainá, A.H.B. 1993. Vainas secas de Acacia sieberiana como suplemento a una dieta forrajera de baja calidad para corderos en crecimiento en el norte de Nigeria. Trop. Anim. Health Prod., 25: 59-64.

McSweeney, C.S., Palmer, B., McNeill, D.M. and Krause, D.O. 2005. Microbial interactions with tannins: nutritional consequences for ruminants. Anim. Feed Sci. Techn., 91: 83-93.

Minson, J.D. 1990. Forage in ruminant nutrition. Academic Press. San Diego.

Min, B.R., Barry, T.N., Attwood, G.T. and McNabb, W.C. 2003. The effect of condensed tannins on the nutrition and health of ruminants fed fresh temperate forages: a review. Anim. Feed Sci. Techn., 106: 3-19.

Nantoumé, H., Forbes, T.D., Hensarling, C. and 


\section{VELÁZQUEZ, GONZÁLEZ, PEREZGROVAS, BÓRQUEZY DOMÍNGUEZ}

Sieckenius, S.S. 2001. Nutritive value and palatability of guajillo (Acacia berlandieri) as a component of goat diets. Small Rum. Res., 40: 139-148.

NRC. 2007. Nutritional requirements of sheep. $5^{\text {th }}$ ed. Natl. Acad. Sci. Washington, D.C.

Parra, V.M.R., 1989. El subdesarrollo agrícola en los Altos de Chiapas. Colección cuadernos universiarios $\mathrm{n}^{\circ} 18$. Universidad Autónoma de Chapingo. Centro de Investigaciones ecológicas del Sureste. San Cristobal de las Casas. Chiapas. México.

Perezgrovas, G.R. 1998. Colaborando para el cambio. Pastoras tzotziles de chiapas participan en el diseño de estrategias de desarrollo. San Cristobal de las Casas, Chiapas. México. Anuario de Estudios Indígenas. UNACH., 7: 351-357.

Perezgrovas, G.R. 1999. Los carneros de San Juan. Ovinocultura Indígena en los altos de chiapas. $2^{\mathrm{a}}$ ed. Instituto de Estudios Indígenas. Universidad Autónoma de Chiapas. San Cristobal de las Casas. Chiapas. México. 75 pp.

Perezgrovas, G.R. 2005. La lana del Tunim Chij el venado de algodón. $1^{\mathrm{a}} \mathrm{ed}$. Instituto de Estudios Indígenas-UNACH. San Cristobal de las Casas. Chiapas. México. 63 pp.

Perezgrovas G.R. y Castro, G.H. 2000a. Producción y calidad de lana en el borrego Chiapas como resultado de selección empírica por pastoras indígenas del sur de México. $\vee$ Congreso Iberoamericano de Razas Autóctonas y CrioIlas. La Habana. Cuba.

Perezgrovas G.R. y Castro, G.H. 2000b. El borrego Chiapas y el sistema tradicional de manejo de ovinos entre las pastoras tzotziles. Arch. Zootec., 49: 391-403.

Perezgrovas, G.R., Guarín, E., Castro, H. y Parry, A. 1997. Producción del vellón sucio y crecimiento de lana en el borrego Chiapas. Congreso Nacional de Producción Ovina. 15 pp.

Seigler, D.S. 2003. Proceedings of the phytochemistry and legume/animal interaction. International Legume Conference in Canberra. Australia. Bioch. System. Ecol., 31: 845-873.

Sen, A.R., Shinde, A.K., Verma, D.L. and Karim, S.A. 2002. Growth carcass traits of Sirohi finisher kids fed on ad lib tree leaves and concentrate. Indian. J. Anim. Sci., 72: 11791181.

Silanikove, N., Gilboa, N., Perevolotsky, A. and Nitzan, Z. 1996. Goats fed tannins-containing leaves do not exhibit toxic syndromes. Small Rum. Res., 21: 195-201.

Steel, R.G.D. and Torrie, J.H. 1980. Principles and procedures of statistics. New York. USA.

Van Keulen, J. and Young, B.A. 1977. Evaluation of acid-insoluble ash as a natural marker in ruminant digestibility studies. J. Anim. Sci., 44: 282-287.

Velázquez, A.J., Perezgrovas, G.R., Velasco, Z.M., Zaragoza, M.L. y Rodríguez, G.G. 2005. Evaluación de vainas de quebracho (Acacia farnesiana) en alimentación de ganado lanar. Arch. Zootec., 54: 535-540.

Velázquez, A.J.,Borquez, C.J., Domínguez, V.I., Perezgrovas, G.R. and González-Ronquillo, M. 2011. Chemical composition and in vitro gas production of diets on varied levels of Acacia farnesiana pods. Arch. Zootec., 60: 637-645.

Archivos de zootecnia vol. 60, núm. 231, p. 488. 